\title{
PERFORMANCE ANALYSIS OF THE CONVENTIONAL COMPLEX LMS AND AUGMENTED COMPLEX LMS ALGORITHMS
}

\author{
Scott C. Douglas
Department of Electrical Engineering
Southern Methodist University
Dallas, Texas 75275 USA

\begin{abstract}
Recently, the augmented complex LMS (ACLMS) algorithm has been proposed for modeling complex-valued signal relationships in which a widely-linear model can be more appropriate [1]. It is not clear, however, how the behavior of ACLMS differs from that of the conventional complex LMS (CCLMS) algorithm. In this paper, we leverage a recently-developed analysis for the complex LMS algorithm [2] to illuminate the performance relationships between the ACLMS and CCLMS algorithms. Our analysis shows that the ACLMS algorithm can potentially achieve a lower steadystate mean-squared error as compared to that of CCLMS, but the convergence speed of ACLMS is slowed in the presence of highly non-circular complex-valued input signals. An adaptive beamforming example indicates the utility of the results.
\end{abstract}

Index Terms - adaptive arrays, adaptive filters, adaptive signal processing, adaptive systems, least mean square methods

\section{INTRODUCTION}

The complex LMS (CLMS) algorithm extends the well-known realvalued LMS algorithm to allow the processing of complex-valued signals found in applications ranging from wireless communications to medicine $[3,4]$. The complex LMS algorithm is given by

$$
\begin{aligned}
y(k) & =\underline{\mathbf{x}}^{H}(k) \underline{\mathbf{w}}(k) \\
e(k) & =d(k)-y(k) \\
\underline{\mathbf{w}}(k+1) & =\underline{\mathbf{w}}(k)+\mu e(k) \underline{\mathbf{x}}(k) .
\end{aligned}
$$

where $\underline{\mathbf{w}}(k)=\left[w_{1}(k) \cdots w_{L}(k)\right]^{T}$ is the weight vector at time $k$, $\underline{\mathbf{x}}(k)=\left[x_{1}(k) \cdots x_{L}(k)\right]^{T}$ is the input signal vector, $y(k)$ is the output signal, $d(k)$ is the desired response signal, $e(k)$ is the error signal, and $\mu$ is the step size.

In the CLMS algorithm, how $\mathbf{x}(k)$ is defined determines the modeling capabilities of the adaptive filter. If $d(n)$ can be wellmodeled from a set of complex measurements $\left\{z_{1}(k), z_{2}(k), \ldots\right.$, $\left.z_{M}(k)\right\}$, then it makes sense to define $\underline{\mathbf{x}}(k)=\mathbf{z}(k)=\left[z_{1}(k) \cdots\right.$ $\left.z_{M}(k)\right]^{T}$ and $L=M$. For this particular choice, $y(k)$ is related to $\mathbf{z}(k)$ through a complex linear model, and we shall employ the algorithm notation $\underline{\mathbf{w}}(k)=\mathbf{h}(k)=\left[h_{1}(k) \cdots h_{M}(k)\right]^{T}$, such that

$$
\begin{aligned}
y(k) & =\mathbf{z}^{H}(k) \mathbf{h}(k) \\
\mathbf{h}(k+1) & =\mathbf{h}(k)+\mu e(k) \mathbf{z}(k) .
\end{aligned}
$$

We shall refer to this procedure as the conventional CLMS (CCLMS) algorithm. Recently, it has been shown that additional modeling capabilities can be obtained by using a widely linear model in which

$$
\begin{gathered}
\underline{\mathbf{x}}(k)=\left[\mathbf{z}^{T}(k) \mathbf{z}^{H}(k)\right]^{T} \\
\underline{\mathbf{w}}(k)=\left[\mathbf{h}^{T}(k) \mathbf{g}^{T}(k)\right]^{T},
\end{gathered}
$$

\author{
Danilo P. Mandic
}

\author{
Department of Electrical and Electronic Engineering \\ Imperial College \\ London, SW7 2BT United Kingdom
}

where $\mathbf{g}(k)=\left[g_{1}(k) \cdots g_{M}(k)\right]^{T}$ and $L=2 M$. With this choice, the system output and coefficient updates are

$$
\begin{aligned}
y(k) & =\mathbf{z}^{T}(k) \mathbf{g}(k)+\mathbf{z}^{H}(k) \mathbf{h}(k) \\
\mathbf{g}(k+1) & =\mathbf{g}(k)+\mu e(k) \mathbf{z}^{*}(k) \\
\mathbf{h}(k+1) & =\mathbf{h}(k)+\mu e(k) \mathbf{z}(k) .
\end{aligned}
$$

We shall refer to this procedure as the augmented CLMS (ACLMS) algorithm.

The behavior of the ACLMS algorithm has been explored through application to specific complex-valued data sets [1]. Particular attributes of its general performance, however, have not been studied. The main challenge in this understanding is in carefully handling the potentially non-circular nature of $\mathbf{z}(k)$, as ACLMS algorithm performance depends on both the covariance matrix $\mathbf{R}_{\mathbf{z}}$ and pseudo-covariance matrix $\mathbf{P}_{\mathbf{z}}$ for $\mathbf{z}(k)$, defined as

$$
\begin{aligned}
\mathbf{R}_{\mathbf{z}} & =E\left\{\mathbf{z}(k) \mathbf{z}^{H}(k)\right\} \\
\text { and } \mathbf{P}_{\mathbf{z}} & =E\left\{\mathbf{z}(k) \mathbf{z}^{T}(k)\right\}
\end{aligned}
$$

respectively, as well as the potential widely-linear relationship between $d(k)$ and $\mathbf{z}(k)$ where a model of the form in (8) may be more appropriate than that in (4).

In this paper, we leverage a recently-developed analysis of the CLMS algorithm for non-circular complex Gaussian random processes [2] as well as other derivations to develop an analytical understanding of and performance comparisons between the CCLMS and ACLMS algorithms. Our results make use of important tools in the independent component analysis literature [5] that allow the joint decomposition of both $\mathbf{R}_{\mathbf{z}}$ and $\mathbf{P}_{\mathbf{z}}$ to enable a careful comparison of the ACLMS and CCLMS algorithms. From these results, several important issues are highlighted, including:

- Unlike the CCLMS algorithm, the ACLMS algorithm converges more slowly when the input signal vector $\mathbf{z}(k)$ is more non-circular.

- The ACLMS algorithm often achieves a lower steady-state MSE due to its additional modeling capability. The steadystate MSE depends both on the degree of input signal noncircularity and on how widely-linear the input-desired response signal relationship is.

An application to adaptive beamforming shows the predictive accuracy and utility of our results and conclusions.

\section{CONVERGENCE SPEED ANALYSIS}

From [2], an analysis of the mean and mean-square behavior of the CLMS algorithm for complex non-circular Gaussian signals has 
been performed. Since both CCLMS and ACLMS are special cases of CLMS, we can leverage the general results in [2] to understand and compare the behaviors of these two algorithms. Only the mean behavior is considered below for brevity, and only general results are outlined due to their similarity to those of other adaptive algorithm analyses. Our study assumes without loss of generality that $d(k)$ is generated from the linear model

$$
d(k)=\mathbf{z}^{T}(k) \mathbf{g}_{o}+\mathbf{z}^{H}(k) \mathbf{h}_{o}+\eta(k),
$$

where $\eta(k)$ is complex non-circular Gaussian and uncorrelated with $\mathbf{x}(k)$, because of the joint Gaussianity of $d(k)$ and $\mathbf{z}(k)$. It also makes use of the well-known independence assumptions that assume that $\{d(l), \mathbf{z}(l)\}$ is independent of $\{d(k), \mathbf{z}(k)\}$ when $k \neq l$.

For the CCLMS algorithm, define the coefficient error vector

$$
\mathbf{v}(k)=\mathbf{h}(k)-\mathbf{h}_{\text {opt }} .
$$

where $\mathbf{h}_{\text {opt }}$ is the steady-state value of $\mathbf{h}(k)$ at convergence for this algorithm. Then, using the general results of [2], the evolutionary behavior of $E\{\mathbf{v}(k)\}$ for CCLMS evolves as

$$
E\{\mathbf{v}(k+1)\}=\left(\mathbf{I}-\mu \mathbf{R}_{\mathbf{z}}\right) E\{\mathbf{v}(k)\} .
$$

For the ACLMS algorithm, define the coefficient error vector

$$
\underline{\mathbf{v}}(k)=\left[\begin{array}{l}
\mathbf{h}(k)-\mathbf{h}_{0} \\
\mathbf{g}(k)-\mathbf{g}_{0}
\end{array}\right]
$$

Then, using the general results of [2], the evolutionary behavior of $E\{\underline{\mathbf{v}}(k)\}$ for ACLMS evolves as

$$
\begin{aligned}
E\{\underline{\mathbf{v}}(k+1)\} & =\left(\mathbf{I}-\mu \mathbf{R}_{\mathbf{x}}\right) E\{\underline{\mathbf{v}}(k)\} \\
\mathbf{R}_{\mathbf{x}} & =\left[\begin{array}{ll}
\mathbf{R}_{\mathbf{z}} & \mathbf{P}_{\mathbf{z}} \\
\mathbf{P}_{\mathbf{z}}^{*} & \mathbf{R}_{\mathbf{z}}^{*}
\end{array}\right]
\end{aligned}
$$

Comparing (15) and (18), both relations have the same form, and these relations match the mean behavior of the real-valued LMS algorithm. As such, the time constant of the $i$ th mode of convergence of the coefficient error is inversely proportional to the $i$ th eigenvalue of $\mathbf{R}_{\mathbf{z}}$ for the CCLMS algorithm and the $i$ th eigenvalue of $\mathbf{R}_{\mathbf{x}}$ for the ACLMS algorithm. Moreover, convergence will be difficult to tune via the choice of $\mu$ in situations where the spread of eigenvalues is large [3]. From these facts, it is clear that, in order for a more detailed performance comparison, convenient eigendecompositions of both $\mathbf{R}_{\mathrm{z}}$ and $\mathbf{R}_{\mathrm{x}}$ are required. We can employ the strong-uncorrelating transform (SUT) for the eigendecomposition of $\mathbf{R}_{\mathbf{x}}$. The SUT is the unique complex matrix $\mathbf{C}$ such that [5]

$$
\begin{aligned}
& \mathbf{R}_{\mathbf{z}}=\mathbf{C} \mathbf{C}^{H}=\mathbf{Q} \boldsymbol{\Sigma}^{2} \mathbf{Q}^{H} \\
& \mathbf{P}_{\mathbf{z}}=\mathbf{C} \boldsymbol{\Lambda} \mathbf{C}^{T}
\end{aligned}
$$

where $\Lambda$ is a diagonal matrix of real-valued non-circularity coefficients $\lambda_{i}, 1 \geq \lambda_{1} \geq \cdots \geq \lambda_{M} \geq 0$, and we have used the complex SVD of $\mathbf{C}$ to represent $\mathbf{R}_{\mathbf{z}}$ where

$$
\mathbf{C}=\mathbf{Q} \boldsymbol{\Sigma} \mathbf{V}^{H} .
$$

Using the SUT, it is possible to decompose $\mathbf{R}_{\mathrm{x}}$ uniquely as

$$
\mathbf{R}_{\mathbf{x}}=\left[\begin{array}{cc}
\mathbf{C} & \mathbf{0} \\
\mathbf{0} & \mathbf{C}^{*}
\end{array}\right]\left[\begin{array}{cc}
\mathbf{I} & \mathbf{\Lambda} \\
\mathbf{\Lambda} & \mathbf{I}
\end{array}\right]\left[\begin{array}{cc}
\mathbf{C}^{H} & \mathbf{0} \\
\mathbf{0} & \mathbf{C}^{T}
\end{array}\right]
$$

Furthermore, using the complex SVD of $\mathbf{C}$, we have

$$
\begin{aligned}
\mathbf{R}_{\mathbf{x}}= & {\left[\begin{array}{cc}
\mathbf{Q} & \mathbf{0} \\
\mathbf{0} & \mathbf{Q}^{*}
\end{array}\right]\left[\begin{array}{cc}
\boldsymbol{\Sigma} & \mathbf{0} \\
\mathbf{0} & \mathbf{\Sigma}
\end{array}\right]\left[\begin{array}{cc}
\mathbf{V}^{H} & \mathbf{0} \\
\mathbf{0} & \mathbf{V}^{T}
\end{array}\right]\left[\begin{array}{cc}
\mathbf{I} & \mathbf{\Lambda} \\
\mathbf{\Lambda} & \mathbf{I}
\end{array}\right] } \\
& \times\left[\begin{array}{cc}
\mathbf{V} & \mathbf{0} \\
\mathbf{0} & \mathbf{V}^{*}
\end{array}\right]\left[\begin{array}{cc}
\mathbf{\Sigma} & \mathbf{0} \\
\mathbf{0} & \mathbf{\Sigma}
\end{array}\right]\left[\begin{array}{cc}
\mathbf{Q}^{H} & \mathbf{0} \\
\mathbf{0} & \mathbf{Q}^{T}
\end{array}\right]
\end{aligned}
$$

In addition, we have that

$\left[\begin{array}{cc}\mathbf{I} & \boldsymbol{\Lambda} \\ \boldsymbol{\Lambda} & \mathbf{I}\end{array}\right]=\frac{1}{\sqrt{2}}\left[\begin{array}{cc}\mathbf{I} & -\mathbf{I} \\ \mathbf{I} & \mathbf{I}\end{array}\right]\left[\begin{array}{cc}\mathbf{I}+\boldsymbol{\Lambda} & \mathbf{0} \\ \mathbf{0} & \mathbf{I}-\boldsymbol{\Lambda}\end{array}\right]\left[\begin{array}{cc}\mathbf{I} & \mathbf{I} \\ -\mathbf{I} & \mathbf{I}\end{array}\right] \frac{1}{\sqrt{2}}(24)$

Thus, the autocorrelation matrix that determines the mean behavior of ACLMS has the following structure:

$$
E\left\{\mathbf{x}(k) \mathbf{x}^{H}(k)\right\}=\mathbf{Q}_{1} \mathbf{D}_{c}^{1 / 2} \mathbf{Q}_{2} \mathbf{D}_{n c} \mathbf{Q}_{2}^{H} \mathbf{D}_{c}^{1 / 2} \mathbf{Q}_{1}^{H},
$$

where $\mathbf{Q}_{1}$ and $\mathbf{Q}_{2}$ are particular complex orthonormal matrices and

$$
\begin{aligned}
\mathbf{D}_{c}^{1 / 2} & =\mathbf{I} \otimes \boldsymbol{\Sigma} \\
\mathbf{D}_{n c} & =\left[\begin{array}{cc}
\mathbf{I}+\boldsymbol{\Lambda} & \mathbf{0} \\
\mathbf{0} & \mathbf{I}-\boldsymbol{\Lambda}
\end{array}\right]
\end{aligned}
$$

We can now compare the convergence behavior of the update relations in (15) and (18), as the eigenstructure of both $\mathbf{R}_{\mathbf{z}}$ and $\mathbf{R}_{\mathbf{x}}$ are completely specified by (19) and (25), respectively.

Remark \#1: As is the case for the real-valued LMS algorithms, the convergence speeds of both CCLMS and ACLMS are exponential, with modes of convergence that depend on the eigenvalues of the $(M \times M)$ matrix $\mathbf{R}_{\mathbf{z}}$ and the the $(2 M \times 2 M)$ matrix $\mathbf{R}_{\mathbf{x}}$, respectively. As in real-valued LMS, an increased eigenvalue spread typically leads to slower convergence for a fixed step size $\mu$ for both algorithms.

Remark \#2: The mean behavior of ACLMS is jointly determined by the eigenvalues $\sigma_{i}^{2}$ of $\mathbf{R}_{\mathbf{z}}$ and the non-circularity coefficients $\lambda_{i}$ of $\mathbf{P}_{\mathbf{z}}$. The way in which these terms combine, however, is nontrivial, as can be seen in (25), such that it is not possible to easily see how a particular distribution of $\left\{\sigma_{i}, \lambda_{i}\right\}$ affects overall convergence performance.

Remark \#3: The worst-case eigenvalue spread of $\mathbf{R}_{\mathbf{x}}$ for the ACLMS algorithm is increased over that of $\mathbf{R}_{\mathbf{z}}$ in the CCLMS algorithm. The condition number or ratio of maximum to minimum eigenvalues of $\mathbf{R}_{\mathbf{z}}$ for the CCLMS algorithm with $M$ taps is

$$
\kappa\left(\mathbf{R}_{\mathbf{z}}\right)=\frac{\sigma_{\max }^{2}}{\sigma_{\min }^{2}} .
$$

The condition number of $\mathbf{R}_{\mathbf{x}}$ for the ACLMS algorithm with $2 M$ taps is upper bounded by

$$
\kappa\left(\mathbf{R}_{\mathbf{x}}\right) \leq \frac{\left(1+\lambda_{\max }\right)}{\left(1-\lambda_{\max }\right)} \kappa\left(\mathbf{R}_{\mathbf{z}}\right) .
$$

So, the convergence speed disparity between the fastest and slowest modes of ACLMS can be much greater than that of CCLMS, particularly if the data is highly non-circular. This fact shows a drawback of ACLMS: with an increased modeling capability comes a potential slowdown in convergence depending on the data statistics. This slowdown is not due to simply having more filter coefficients to adapt; it is structural within the algorithm itself. 
Remark \#4: An easily computed step size bound for ACLMS something that is extremely important from a practical perspective - can be found from the mean analysis:

$$
0<\mu<\frac{1}{\operatorname{tr}\left[\mathbf{R}_{\mathbf{z}}\right]}
$$

Thus, the upper bound on the step size for ACLMS is roughly half the value one might use for CCLMS.

\section{STEADY-STATE MEAN-SQUARE ERROR ANALYSIS}

The CCLMS and ACLMS algorithms have differing modeling capabilities due to the fundamental difference in their linear input-output models as indicated in (4) and (8), respectively. As a result, the mean-square performances of the algorithms differ in steady-state. In this section, we determine the difference in steady-state MSE assuming that both algorithms have converged. We neglect any performance differences due to errors in adaptation caused by a non-zero step size, such as excess MSE or misadjustment in steady-state. We assume that $d(k)$ is generated according to the widely-linear model in (13), where both $\mathbf{z}(k)$ and $\eta(k)$ are jointly Gaussian with zero means and covariances $\mathbf{R}_{\mathbf{z}}$ and $\sigma_{\eta}^{2}$, respectively. Moreover, we assume that $E\{\eta(k) \mathbf{z}(k)\}=\mathbf{0}$.

For the ACLMS algorithm, the minimum MSE achievable is given by

$$
\xi_{M M S E}^{(A C L M S)}=\min _{\mathbf{g}, \mathbf{h}} E\left\{\left|d(k)-\mathbf{z}^{T}(k) \mathbf{g}-\mathbf{z}^{H}(k) \mathbf{h}\right|^{2}\right\}(31)
$$

This minimum is achieved when $\mathbf{g}=\mathbf{g}_{o}$ and $\mathbf{h}=\mathbf{h}_{o}$, such that

$$
\xi_{M M S E}^{(A C L M S)}=\sigma_{\eta}^{2} .
$$

For the CCLMS algorithm, the optimum solution for $\mathbf{h}(k)$ is not necessarily equal to $\mathbf{h}_{o}$ due to the widely-linear nature of the relationship between $\mathbf{z}(k)$ and $d(k)$. Thus, we must employ the classic expressions for minimum MSE estimation, in which

$$
\mathbf{h}_{\text {opt }}=\mathbf{R}_{\mathbf{z}}^{-1} E\{d(k) \mathbf{z}(k)\} .
$$

It is straightforward to show that

$$
E\{d(k) \mathbf{z}(k)\}=\mathbf{R}_{z} \mathbf{h}_{o}+\mathbf{P}_{z} \mathbf{g}_{o},
$$

such that

$$
\mathbf{h}_{\text {opt }}=\mathbf{h}_{o}+\mathbf{R}_{\mathbf{z}}^{-1} \mathbf{P}_{z} \mathbf{g}_{o} .
$$

The minimum MSE for the CCLMS algorithm is therefore

$$
\xi_{M M S E}^{(C C L M S)}=E\left\{\left|d(k)-\mathbf{z}^{H}(k) \mathbf{h}_{o}-\mathbf{z}^{H}(k) \mathbf{R}_{\mathbf{z}}^{-1} \mathbf{P}_{z} \mathbf{g}_{o}\right|^{2}\right\}(36)
$$

which after expanding this relation and evaluating all expectations results in

$$
\xi_{M M S E}^{(C C L M S)}=\xi_{M M S E}^{(A C L M S)}+\mathbf{g}^{H}\left[\mathbf{R}_{\mathbf{z}}^{*}-\mathbf{P}_{\mathbf{z}}^{*} \mathbf{R}_{\mathbf{z}}^{-1} \mathbf{P}_{\mathbf{z}}\right] \mathbf{g}_{o}
$$

From these results, several remarks are in order:

Remark \#5: The matrix $\mathbf{R}_{\mathbf{z}}^{*}-\mathbf{P}_{\mathbf{z}}^{*} \mathbf{R}_{\mathbf{z}}^{-1} \mathbf{P}_{\mathbf{z}}$ in (37) is known as the Schur complement of $\mathbf{R}_{\mathrm{x}}$ [6]. Because $\mathbf{R}_{\mathrm{x}}$ is positive semi-definite, the matrix $\mathbf{R}_{\mathbf{z}}^{*}-\mathbf{P}_{\mathbf{z}}^{*} \mathbf{R}_{\mathbf{z}}^{-1} \mathbf{P}_{\mathbf{z}}$ is also positive semi-definite. Hence,

$$
\xi_{M M S E}^{(C C L M S)} \geq \xi_{M M S E}^{(A C L M S)}
$$

due to the quadratic form appearing on the right of (37). Thus, the minimum MSE of the augmented complex LMS algorithm will be no greater than that of the conventional complex LMS algorithm.

Remark \#6: The minimum MSEs of ACLMS and CCLMS are the same if at least one of the following two conditions holds:
1. The system being modeled is complex linear, e.g. $\mathbf{g}_{o}=\mathbf{0}$.

2. The complex signal $\mathbf{z}(k)$ has a real-valued subspace where the circularity coefficients $\lambda_{i}$ are unity and $\mathbf{g}_{o}$ falls entirely within this subspace, such that $\left[\mathbf{R}_{\mathbf{z}}^{*}-\mathbf{P}_{\mathbf{z}}^{*} \mathbf{R}_{\mathbf{z}}^{-1} \mathbf{P}_{\mathbf{z}}\right] \mathbf{g}_{o}=\mathbf{0}$.

As the second condition is highly-unlikely in practice, CCLMS will be competitive with ACLMS only in situations involving complexlinear models.

Remark \#7: If the input signal $\mathbf{z}(k)$ is circular such that $\mathbf{P}_{\mathbf{z}}=\mathbf{0}$, the minimum MSE of CCLMS can still be larger than that of ACLMS if the relationship between $\mathbf{z}(k)$ and $d(k)$ is widely-linear. This result points to the lack of any connection between the non-circularity of $\mathbf{z}(k)$ and the widely-linear nature of the input-output model; that is, circularly-distributed data does not imply that a complex linear model is appropriate.

Remark \#8: If the relationship between $\mathbf{z}(k)$ and $d(k)$ is complex linear, then the minimum MSEs of ACLMS and CCLMS are the same. In such cases, one should choose CCLMS over ACLMS based on the results of the last section, as ACLMS may converge more slowly despite achieving a similar MSE in steady-state due to signal non-circularity.

\section{SIMULATIONS}

We now compare the behaviors of the CCLMS and ACLMS algorithms in a useful signal processing task: beamforming for multiport antenna arrays. Such systems exploit spatial diversity to build one or more signal estimates from a set of measured signals. For a uniform linear array (ULA), an appropriate signal model for a set of received signals $\left\{z_{i}(k)\right\}, 1 \leq i \leq M$ is

$$
\begin{aligned}
z_{i}(k) & =\nu_{i}(k)+\sum_{p=1}^{N} \delta_{i}\left(\phi_{p}\right) s_{p}(k) \\
\delta_{i}(\phi) & =\exp \left(-j 2 \pi \frac{\Delta \sin (\phi)}{\gamma}(i-1)\right)
\end{aligned}
$$

where $s_{p}(k)$ is the signal from the $p$ th user at time $k, \phi_{p}$ is the angle of arrival of the $p$ th narrowband signal with respect to the array normal, $\Delta$ is the inter-element antenna spacing, $\gamma$ is the wavelength, and $\nu_{i}(k)$ is the complex-valued sensor noise at the $i$ th sensor. Using either (4) or (8), an output signal can be produced to estimate one of the user signals $s_{p}(k)$. Parallel versions of this structure are used to estimate several user signals simultaneously.

For our simulations, the normalized sensor spacing is $\Delta / \gamma=$ $1 / 2$, the number of sources is $N=4$, and the number of sensor elements is $M=3$. Because the number of sources is smaller than the number of sensors, it is not possible to resolve each source using a traditional complex beamformer adapted using the CCLMS algorithm. It is possible, however, to resolve all four sources using $y(k)$ in (8) if if two or more of the sources are real-valued. Let $s_{1}(k)$ and $s_{2}(k)$ be two independent BPSK signals, and let $s_{3}(k)$ and $s_{4}(k)$ be two independent QPSK signals. Furthermore, let $\left\{\phi_{1}, \phi_{2}, \phi_{3}, \phi_{4}\right\}=\left\{-45^{\circ}, 8^{\circ},-13^{\circ}, 30^{\circ}\right\}$, and let the complex Gaussian measurement noise be of such a level that the signal-tonoise ratio (SNR) of each source in each sensor is $25 \mathrm{~dB}$. We apply four versions of the CCLMS algorithm and four versions of the ACLMS algorithm to this data, with $d(k)=s_{p}^{*}(k), 1 \leq p \leq 4$, and $\mu=0.0001$. Shown in Fig. 1 are the baseband output signal constellations for $4500 \leq k \leq 5000$ for the ACLMS beamformers, in which the BPSK and QPSK output signal patterns are readily 

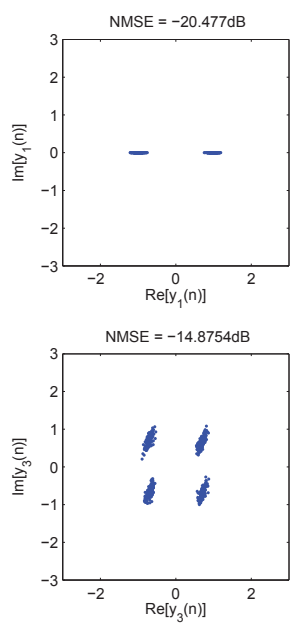
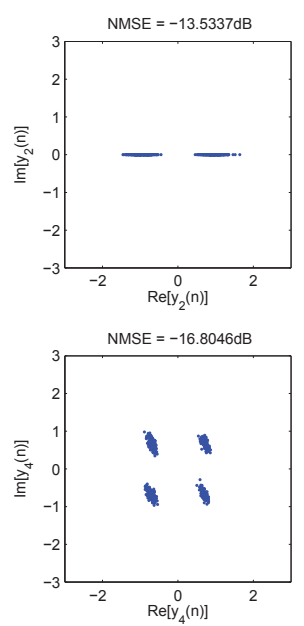

Fig. 1. Output signal constellations for the ACLMS beamformers.
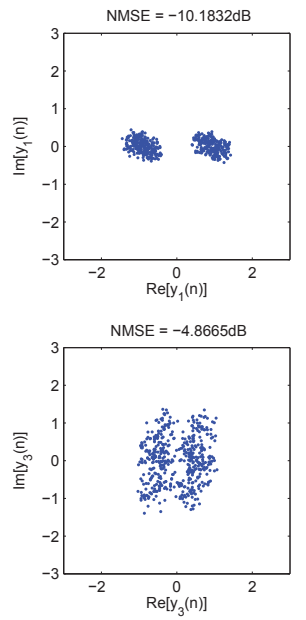
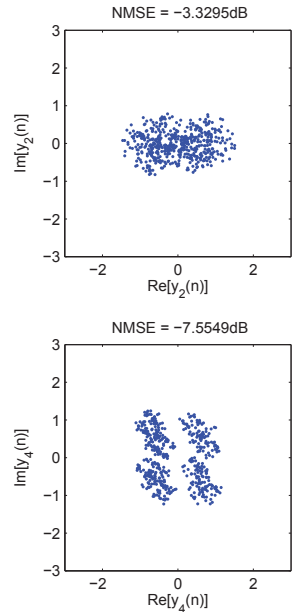

Fig. 2. Output signal constellations for the CCLMS beamformers.

evident. The normalized mean-square errors (NMSEs) are close to the true values computed from theory, given by $-21.045,-14.145$, -15.510 , and $-17.381 \mathrm{~dB}$, respectively. Fig. 2 shows the corresponding performance for the tranditional CCLMS beamformers, in which the BPSK and QPSK output signal patterns are not resolved for sources 2 and 3 and are barely resolvable for sources 1 and 4 . The NMSEs for these beamformers are also higher than those for the ACLMS beamformers.

Figs. 3 and 4 show the convergence of the squared errors for these simulations for the ACLMS and CCLMS algorithms, respectively. From these plots, it is readily apparent that the ACLMS algorithm converges much more slowly - about 2500 iterations - than does the CCLMS algorithm - about 50 iterations - for this data. This fact could have been easily determined from the condition numbers $\kappa\left(\mathbf{R}_{\mathbf{z}}\right)=117.1$ and $\kappa\left(\mathbf{R}_{\mathbf{x}}\right)=2.618$, indicating that the worstcase convergence rate of the ACLMS algorithm will be 45 times slower than that of the CCLMS algorithm for similar fast-converging modes. Also shown are the minimum MSEs for all of the beamformers as a dashed line, indicating that the algorithms' MSEs do approach these minimum MSEs.
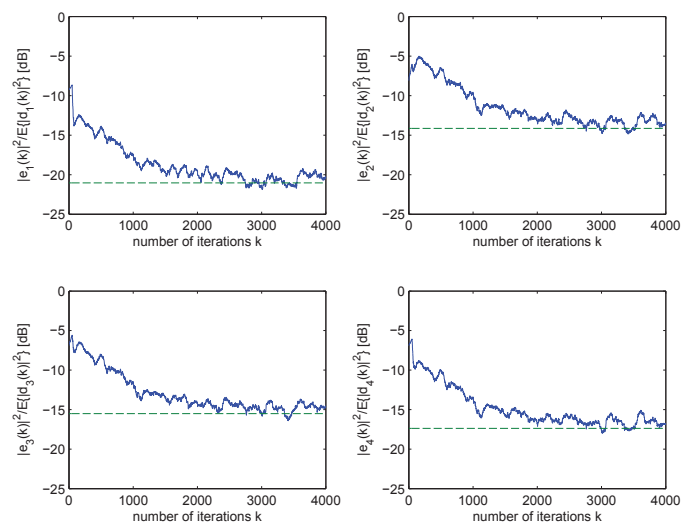

Fig. 3. Convergence of the errors for the ACLMS beamformers.
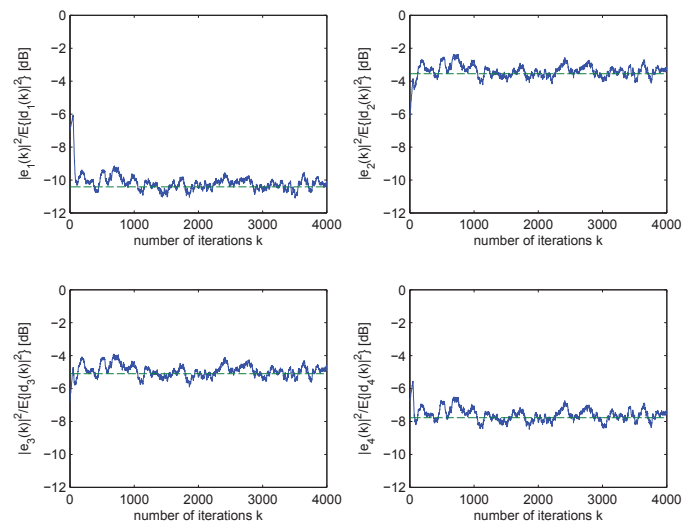

Fig. 4. Convergence of the errors for the CCLMS beamformers.

\section{CONCLUSIONS}

In this paper, we have examined the performance relationships between the conventional and augmented CLMS algorithms for modeling complex-valued systems that are potentially widely-linear and driven by signals with potentially non-circular complex statistics. Our study indicates that the ACLMS can achieve a lower steady-state MSE due to its additional modeling capabilities, but its convergence speed is likely to be slower than that of the CCLMS algorithm. These attributes are illustrated in an adaptive beamforming example. Additional results concerning the mean-square performance of ACLMS and CCLMS and the interplay of modeling capability vs. excess mean-square error is the subject of current investigation.

\section{REFERENCES}

[1] S. Javidi, M. Pedzisz, S.L. Goh, and D.P. Mandic, "The augmented complex leas mean square algorithm with application to adaptive prediction problems", Proc. IAPR Workshop Cognitive Information Processing Syst., Santorini, Greece, Session P, June 2008

[2] S.C. Douglas and D.P. Mandic, "Mean and mean-square analysis of the complex LMS algorithm for non-circular Gaussian signals," submitted.

[3] B. Widrow, J. McCool, and M. Ball, "The complex LMS algorithm," Proc. IEEE, vol. 63 , pp. 719-720, Apr. 1975.

[4] N.J. Bershad, "On the real and complex least mean square adaptive filter algorithms," Proc. IEEE, vol. 69, pp. 469-470, Apr. 1981.

[5] J. Eriksson and V. Koivunen, "Complex random vectors and ICA models: identifiability, uniqueness, and separability," IEEE Trans. Inform. Theory, vol. 52, pp. 1017-1029, Mar. 2006

[6] T. Kailath, Linear Systems (Englewood Cliffs, NJ: Prentice-Hall, 1980). 